\title{
A Comparative Study of Oral Clonidine and Oral Midazolam as Premedicants for General Anaesthesia
}

\author{
Amitayu Nandi ${ }^{1}$, Purba Haldar², Arup Ghosh³ ${ }^{3}$ Supriya Bhattacharya ${ }^{4}$, Manisankar Nath ${ }^{5}$, Amit Kumar Hazra ${ }^{6}$ \\ ${ }^{1}$ Department of Anaesthesiology, RGMC\&H, Birbhum, West Bengal, India. ${ }^{2}$ Department of Anaesthesiology, RGMC\&H, \\ Birbhum, West Bengal, India. ${ }^{3}$ Department of Anaesthesiology, RGMC\&H, Birbhum, West Bengal, India. ${ }^{4}$ Department \\ of Anaesthesiology, RGMC\&H, Birbhum, West Bengal, India. ${ }^{5}$ Department of Anaesthesiology, RGMC\&H, Birbhum, \\ West Bengal, India. ${ }^{6}$ Department of Anaesthesiology, RGMC\&H, Birbhum, West Bengal, India.
}

\section{ABSTRACT}

\section{BACKGROUND}

Preanaesthetic medication should be effective and pleasant to be taken orally, have analgesic and non-emetic properties, should not impair cardiovascular stability or depress respiration and produce adequate sedation and anxiolysis. We wanted to compare the effectiveness of oral clonidine and oral midazolam as preanaesthetic medicants.

\section{METHODS}

After obtaining the institutional ethical committee clearance and written informed consent, 60 patients, selected for surgery under general anaesthesia were divided by computer generated randomization in Group C $(n=30)$ : received Tablet clonidine 150 mcg ( $0.15 \mathrm{mg})$ and Group M ( $\mathrm{n}=30)$ : received Tablet midazolam $7.5 \mathrm{mg}$. Noninvasive blood pressure (systolic, diastolic \& mean), respiratory rate, heart rate, degree of sedation, degree of anxiolysis, were recorded at, just before the administration of the any study drug which was 90 minutes before to the induction of anaesthesia, just before to induction of anaesthesia, three minutes after the orotracheal intubation, every 10 minutes for 3 such readings, and three minutes after the orotracheal extubation, and were statistically analysed.

\section{RESULTS}

Oral clonidine produced significant attenuation of systolic, diastolic \& mean arterial pressure, and reduced respiratory rate, than oral midazolam. Oral midazolam was able to attenuate the pulse rate in a better way than oral clonidine. Oral clonidine produced significant sedation and anxiolysis in comparison to patients who receiving oral midazolam.

\section{CONCLUSIONS}

Oral clonidine is the better attenuator amongst the two drugs studied as premedicants to attenuate the cardiovascular responses to laryngoscopy and intubation.

\section{KEY WORDS}

Clonidine, Midazolam, Premedication, Haemodynamic Response, Anxiolytics, Sedation

\author{
Corresponding Author: \\ Dr. Purba Haldar, \\ Flat-L3, Block IV, \\ Swabhumi Residency, \\ P-12, Motijheel Avenue, \\ Kolkata-700074, West Bengal, India. \\ E-mail: purbahaldar@gmail.com
}

DOI: $10.14260 / \mathrm{jemds} / 2020 / 252$

Financial or Other Competing Interests: None.

How to Cite This Article:

Nandi A, Haldar P, Ghosh A, et al. A Comparative study of oral clonidine and oral midazolam as premedicants for general anaesthesia. J. Evolution Med. Dent. Sci. 2020;9(14):1161-1166, DOI: $10.14260 / \mathrm{jemds} / 2020 / 252$

Submission 17-01-2019, Peer Review 13-03-2020

Acceptance 20-03-2020,

Published 06-04-2020.

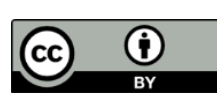




\section{BACKGROUND}

Preanaesthetic medication forms an integral part of anaesthetic management and some form of premedication is universally administered before any anaesthesia. The ideal premedicant should be effective and pleasant to be taken orally, have analgesic and non-emetic properties, should not impair cardiovascular stability or depress respiration, should have antisialagogue effect and should effectively alleviate apprehension of the patient. ${ }^{1}$ In modern anaesthesia, endotracheal Intubation is essential for balanced anaesthesia and for resuscitative measures in intensive care units. During induction of general anaesthesia two important events take place. One is laryngoscopy, the other one being tracheal intubation. During laryngoscopy, blade of laryngoscope presses against the base of tongue and lifts up the epiglottis. This incidence gives rise to certain impulses resulting in intense sympathetic stimulation causing hypertension and tachycardia. ${ }^{2}$ Compared to endotracheal intubation, laryngoscopy causes more intense stimulation so far as cardiovascular effects are concerned. Thus the stimuli produced by laryngoscopy and intubation carried through the afferent pathway through the glossopharyngeal and branches of vagus while the efferent discharge carrying the cardiovascular responses through the cervical sympathetic nerves. This reflex sympathetic stimulation leads to liberation of catecholamines ${ }^{3}$ which are responsible for changes in cardiovascular parameters. The increase in systolic, diastolic and mean arterial pressure increase the left ventricular workload and myocardial oxygen consumption. An increase in heart rate is associated with increased cardiac work and reduction in coronary filling during diastole. This imbalance between myocardial oxygen demand and supply leads to myocardial ischemia. The changes usually recorded include a rise of systolic blood pressure [SBP] by about $30-50 \mathrm{~mm} \mathrm{Hg}$, diastolic blood pressure [DBP]-about 20-30 mm $\mathrm{Hg}$, resulting in rise in mean arterial pressure [MAP].

Heart rate increases by about $20-40$ beats per minute. Various cardiac dysrhythmias apart from sinus bradycardia and tachycardia do occur in $5-10 \%$ of the patients. However, most of these are benign and transient. Sympathoadrenal stimulation may prove detrimental to the health to a certain group of patients, e.g. those with ischemic heart disease who may suffer acute myocardial infarction. In patients with inotropically compromised heart such an increase in heart rate may lapse them into heart failure. In the case of patients with cerebral aneurysm may result hypertensive haemorrhage in brain. Therefore to prevent these casualties one must try to attenuate the sympathoadrenal stimulation. This observation led to use of different techniques and drugs to attenuate cardiovascular responses to laryngoscopy and tracheal intubation like deeper plane of anaesthesia, local anaesthetics, ${ }^{4}$ [applied both locally and intravenously], narcotics, 5,6 vasodilators, calcium channel antagonists, beta-1 adrenoreceptor blocker, ${ }^{7}$ alpha- 2 adrenoreceptor agonists, or their combinations with various degrees of success. But no single method has gained widespread acceptance because each method has its own merits and demerits. Many new studies are still being carried out with re-evaluation of older ones. With this idea an endeavour was made to evaluate whether oral clonidine or oral midazolam as a premedicant can modify cardiovascular responses to direct laryngoscopy and endotracheal intubation.

\section{Objectives}

1. To compare effectiveness of oral clonidine and oral midazolam as preanaesthetic medications,

2. To compare the haemodynamic changes, respiratory rate, the degree of sedation, anxiolysis, during and following laryngoscopy and endotracheal intubation,

3. To observe any undesirable effects with both study drugs

4. To find out which study drug is preferable as preanaesthetic medication for general anaesthesia.

\section{METHODS}

After obtaining approval from the Ethical Committee of the institution, a prospective, randomised and double blind study was conducted for one year period. Study participants were selected amongst patients selected for surgery under general anaesthesia requiring endotracheal intubation. Before performing the study, we hypothesised that the beneficial effect of oral midazolam and oral clonidine alone will be evident in at least $25 \%$ of the patients, after reviewing related articles. Considering an absolute improvement in the primary outcome by 40 to $50 \%$ in the study group can be considered as clinically relevant and based on a Type I error level of 0.05 , Type II error level 0.2 and a two-sided test, we needed 25 patients in each group. Therefore, to account for probable dropouts, a number of 30 patients in each group was proposed. 60 patients were randomly divided into 2 groups of 30 each: Group C ( $\mathrm{n}=30)$ : received Tablet clonidine $150 \mathrm{mcg}(0.15 \mathrm{mg})$ per orally and Group M ( $\mathrm{n}=30)$ : received Tablet midazolam 7.5 mg per orally. Patients between 30 to 50 years of age, 40 to 60 kgs of weight of either sex (male or Female)belonging to American Society of Anaesthesiologists physical status (ASAPS) with grade I and grade II, with duration of elective surgery: 45 minutes to 90 minutes done under General anaesthesia with controlled ventilation by orotracheal tube were included in study. The Exclusion Parameters were patients belonging to American Society of Anaesthesiologists physical status (ASAPS) with the grade III, IV and V, any history of allergy, hypersensitivity or contraindication to any of the both study drugs, history of any anticholinergic drug was given before or at the time of induction or during maintenance of anaesthesia. It was a prospective, randomised and double blind study. All the patients were assigned into one of the two groups by using blinded envelopes were prepared with the help of a random number chart. A fellow anaesthesiologist who was not involved in the present study was given the study drug in powder form with sips of water, about 90 minutes prior to the induction of anaesthesia. The investigator and the patients were unaware of the nature of the study drug.

After detailed history, thorough physical examination and necessary preoperative investigations, Informed written consent was taken from the all selected study patients. Tablet Ranitidine (150 mg) and Alprazolam (0,5 mg) were given per orally at the night before the surgery. Nil per orally for 6 to 8 hours prior to surgery. Inj. Ondansetron ( $4 \mathrm{mg}$ ) I/V was given 30 minutes prior to induction. All patients were monitored with standard monitoring technique. After securing I/V access intravenous fluid (ringer's lactate) was started. Inj. Tramadol 
(50 mg) I/V was given slowly. Pre oxygenation for 5 minutes. Induction was done with inj. Thiopentone (4 mg/Kg). Laryngoscopy and orotracheal intubation was done when adequate jaw relaxation was achieved in a single attempt with Inj. Vecuronium $(0.5 \mathrm{mg} / \mathrm{Kg})$. And mechanical ventilation continued. Maintenance of anaesthesia was done with Oxygen \& Nitrous oxide (1:2), inj. Vecuronium $(0.025 \mathrm{mg} / \mathrm{Kg})$ and intermittent Isoflurane inhalation. At the end of surgery patients were reversed adequately and extubated. Assessment of pulse rate (beats/minute), systolic, diastolic \& mean bloods pressure ( $\mathrm{mm} \mathrm{Hg}$ ), degree of sedation (Ramsay sedation score) ${ }^{8}$ and anxiolysis (Hamilton anxiety Rating Scale) ${ }^{9}$ were done at different time period was done by the investigator at seven different time points [Just before the administration of the any study drug which was 90 minutes before to the induction of anaesthesia (1), Just before to induction of anaesthesia (2), three minutes after the orotracheal intubation (3), after that in every 10 minutes for 3 such reading $(4,5,6)$ and three minutes after the orotracheal extubation (7)].

\section{Statistical Analysis}

Analysis was done only after the Intra Operative Patient Record Sheet of the last subject was in, data was entered, and the computer database cleaned and locked. Data were summarized by routine descriptive statistics. Numerical variables were compared between groups by Student's unpaired $t$ test and Mann-Whitney $U$ test, if otherwise. Intragroup comparisons of numerical variables were done by Student's paired t test, Wilcoxon's matched pairs signed rank test and repeated measure's ANOVA followed by Tukey's Multiple Comparison Test. Fisher's exact test was employed for intergroup comparison of categorical variables. All analyses were two-tailed and $\mathrm{p}<0.05$ was taken to be statistically significant. Data was entered in Microsoft Excel database and analyzed with the help of standard statistical software, namely: Statistica version 6 [Tulsa, Oklahoma: Stat Soft Inc., 2001] and Graph Pad Prism version 4 [San Diego, California: Graph Pad Software Inc., 2005].

\section{RESULTS}

Among the total 60 cases 39 were male and 21 were female. The total sex ratio (M: F) is $1.86: 1$. The mean age was $37.7 \pm$ 6.67 in Group C, $40.5 \pm 6.92$ in Group M. Body weight distribution was $53.1 \pm 5.65$ in Group C, $52.8 \pm 5.20$ in Group $\mathrm{M}$. They were comparable regarding age and weight and small difference between those mean ages and weight was found to be statistically insignificant $(p>0.05)$. Patients of group $C$ [Clonidine] were having statistically significant fall in systolic blood pressure (SBP) about 90 minutes after the administration of oral clonidine [time point- 2] $(p<0.01)$. About 3 min. after the laryngoscopy and orotracheal intubation [time point- 3], there was a peak increase of systolic blood pressure in both the groups those were statistically highly significant $(\mathrm{p}<0.001)$. This increase was highest in Group M [8.89 \%] and was lowest in Group C [7.54 \%]. Both clonidine and midazolam produced a statistically significant increase in systolic blood pressure at 13 minutes after intubation [time point- 4]. With clonidine and midazolam, the systolic blood pressure returned to basal value within 23 minutes after intubation [time point- 5]. At 33 minutes after intubation [time point- 6 ] and 3 minutes after extubation [time point- 7] it came down below the basal level which is statistically significant. At time point- 7 maximum decrease was observed in clonidine group. There was a peak increase in diastolic blood pressure (DBP) at the $3 \mathrm{~min}$ after laryngoscopy and intubation [time point- 3] in both groups. This increase was highly significant $[\mathrm{p}<0.001]$ in both the groups when compared to basal value. This peak increase was slightly higher in Group M [9.41 \%], and lower in Group C [9.15 \%]. But there was significant decrease of diastolic blood pressure at the time point - $6 \& 7$ in both Groups. There was a peak increase in mean arterial pressure (MAP) at the 3 min after laryngoscopy and intubation [time point- 3]. This increase was highly significant in both the Groups when compared to basal value $[p<0.001]$. This peak increase was slightly higher in Group M [8.86 \%], and lower in Group C [8.45 \%]. Both clonidine and midazolam produced a significant decrease in mean arterial pressure [MAP] at the time point -6 and 7 which was the statistically significant.

\begin{tabular}{|ccccccc|}
\hline \multicolumn{7}{c|}{ Time Points } \\
Group C & $\mathbf{2}$ & $\mathbf{3}$ & $\mathbf{4}$ & $\mathbf{5}$ & $\mathbf{6}$ & $\mathbf{7}$ \\
Group M & 1.51 & 8.45 & 4.18 & 0.20 & -3.32 & -6.04 \\
\hline Table 1. Percentage (\%) of Mean of Mean Arterial Pressure [MAP] \\
Increase [+] or Decrease [-] in Comparison to Time Point- 1 [Basal \\
Level] in Group C and Group $\mathbf{M}$ \\
\hline
\end{tabular}

Table 1 shows increase in MAP in both the Groups just 3 min. after laryngoscopy and intubation [time point- 3], as almost same in both Group, then a gradual return to baseline. However both in Group C \& M from time points - 6 onwards it was lower than baseline.

Patients of clonidine Group were having significant rise of pulse rate at just before induction and $3 \mathrm{~min}$., $13 \mathrm{~min}$., $23 \mathrm{~min}$. \& $33 \mathrm{~min}$. after laryngoscopy and intubation [time point- 2,3 , $4,5 \& 6]$. These rises were statistically significant. Patients of midazolam Group were having significant rise of pulse rate at just before induction and $3 \mathrm{~min}$., $13 \mathrm{~min}$., \& $23 \mathrm{~min}$. after laryngoscopy and intubation [time points- 2, 3, 4, \& 5]. These rises were statistically significant. There was significant reduction in pulse rate at time point- 7 . Increase in pulse rate in both groups at just before induction and $3 \mathrm{~min} ., 13 \mathrm{~min}$., \& $23 \mathrm{~min}$. after laryngoscopy and intubation [time points- 2, 3, 4, \& 5] was highest in clonidine Group \& lowest in midazolam Group. Thereafter there was a gradual decrease in pulse rate in both the groups \& in midazolam Group decrease from base line value was observed.

\begin{tabular}{|ccccccc|}
\hline \multicolumn{7}{c|}{ Time Points } \\
Group C & $\mathbf{2}$ & $\mathbf{3}$ & $\mathbf{4}$ & $\mathbf{5}$ & $\mathbf{6}$ & $\mathbf{7}$ \\
Group M & 5.52 & 16.19 & 12.55 & 8.78 & 3.89 & 0.38 \\
\hline Table 2. Percentage (\%) of Mean of Pulse Rate [PR] Increase [+] or \\
Decrease [-] in Comparison to Time Point-1 [Basal Level] in Group C \\
and Group M.
\end{tabular}

Table 2 shows increase in pulse rate in both groups at just before induction and $3 \mathrm{~min} ., 13 \mathrm{~min}$., \& $23 \mathrm{~min}$. after laryngoscopy and intubation [time points- $2,3,4, \& 5]$.This was highest in clonidine Group \& lowest in midazolam Group. Thereafter there was a gradual decrease in pulse rate in both 
the groups \& in midazolam Group decrease from base line value was observed.

Patients of group C \& M both were having statistically significant fall in respiratory rate (RR) about 90 minutes after the administration of oral clonidine and midazolam respectively [time point-2] $(\mathrm{p}<0.001)$.

\begin{tabular}{|ccc|}
\hline \multirow{2}{*}{ With Clonidine } & \multicolumn{3}{c|}{ Time Points } \\
& RR1 (Breaths/Min.) & RR2 (Breaths/Min.) \\
Range & $12-26$ & $10-24$ \\
Mean \pm SD & $17.1 \pm 3.48$ & $15.1 \pm 3.44$ \\
SE & 0.64 & 0.63 \\
p value & \multicolumn{2}{c}{ Time Points } \\
With Midazolam & RR1 (Breaths/Min.) & RR2 (Breaths/Min.) \\
Range & $12-24$ & $11-22$ \\
Mean \pm SD & $16.2 \pm 2.99$ & $15.0 \pm 2.92$ \\
SE & 0.55 & 0.53 \\
p value & \multicolumn{3}{c}{0.00032} \\
\hline Table 3. Changes of Respiratory Rate [RR] in the Group C [Clonidine] \\
Patients ( $\mathbf{n}=\mathbf{3 0}$ ) and Group M (Midazolam) at Different Time Points \\
\hline \multicolumn{2}{|c}{} \\
\hline
\end{tabular}

Table 3 shows Patients of group C [Clonidine] were having statistically significant fall in respiratory rate (RR) about 90 minutes after the administration of oral clonidine [time point2]. Patients of group $M$ [Midazolam] were also having statistically significant fall in respiratory rate about 90 minutes after the administration of oral midazolam [time point-2].

Patients of clonidine group were having statistically significant sedation at 90 minutes after the administration of oral clonidine [time point-2] $(\mathrm{p}=0.002)$. Patients of midazolam group were having also sedation at 90 minutes after the administration of oral midazolam [time point-2], but that was statistically insignificant $(\mathrm{p}=0.116)$.

\begin{tabular}{|ccc|}
\hline With Clonidine & \multicolumn{2}{c|}{ Time Points } \\
Range & $0-2$ & SedSco2 \\
Mean \pm SD & $0.5 \pm 0.73$ & $0-3$ \\
Median \pm IQR & $0 \pm 1$ & $1.1 \pm 0.94$ \\
SE & 0.13 & $1 \pm 2$ \\
p value & \multicolumn{2}{c}{ Time points } \\
With Midazolam & SedSco1 & SedSco2 \\
Range & $0-2$ & $0-3$ \\
Mean \pm SD & $0.5 \pm 0.57$ & $0.7 \pm 0.83$ \\
Median \pm IQR & $0 \pm 1$ & $1 \pm 1$ \\
SE & 0.10 & 0.15 \\
p value & \multicolumn{2}{c}{0.116} \\
\hline Table 4. Changes of Sedation Score [SedSco] in the Group C [Clonidine] \\
and Group M (Midazolam) Patients at Different Time Points \\
\hline
\end{tabular}

Table 4 shows Patients of clonidine group were having statistically significant sedation at 90 minutes after the administration of oral clonidine [time point-2]. Patients of midazolam group were having also sedation at 90 minutes after the administration of oral midazolam [time point-2] but that was statistically insignificant.

Patients of clonidine group were having statistically significant anxiolysis at 90 minutes after the administration of oral clonidine [time point-2].Patients of midazolam group were having little anxiolysis at 90 minutes after the administration of oral midazolam [time point-2] but that was statistically insignificant $(p=0.008)$. But Patients of group $M$ were having little anxiolysis at 90 minutes after the administration of oral midazolam [time point-2] and that was statistically insignificant $(\mathrm{p}=0.554)$.

\begin{tabular}{|ccc|}
\hline With Clonidine & \multicolumn{2}{c|}{ Time Points } \\
Range & AnxSco1 & AnxSco2 \\
Mean \pm SD & $0-3$ & $0-2$ \\
Median \pm IQR & $0.8 \pm 0.99$ & $0.4 \pm 0.56$ \\
SE & $1 \pm 1$ & $0 \pm 1$ \\
p value & 0.18 & 0.10 \\
With Midazolam & \multicolumn{2}{c}{ Time points } \\
Range & AnxSco1 & AnxSco2 \\
Mean \pm SD & $0-3$ & $0-2$ \\
Median \pm IQR & $0.7 \pm 0.8$ & $0.6 \pm 0.63$ \\
SE & $0.5 \pm 1$ & $0.5 \pm 1$ \\
p value & 0.15 & 0.11 \\
& \multicolumn{2}{c}{0.554} \\
\hline Table 5. Changes of Anxiety Score [AnxSco] in the Group C [Clonidine] \\
Patients and Group M (Midazolam) Patients at Different Time Points \\
\hline
\end{tabular}

Table 5 shows Patients of clonidine group were having statistically significant anxiolysis at 90 minutes after the administration of oral clonidine [time point-2]. Patients of midazolam group were having little anxiolysis at 90 minutes after the administration of oral midazolam [time point-2] but that was statistically insignificant.

Oral clonidine as preanaesthetic medication was unable to attenuate the pulse rate, but it produced significant attenuation of systolic, diastolic \& mean arterial pressure and reduced respiratory rate too. Oral midazolam as preanaesthetic medication was able to attenuate the pulse rate in a better way than oral clonidine. It produced less significant attenuation of systolic, diastolic and mean arterial pressure throughout the study period. But it reduced respiratory rate significantly. It was also found that oral clonidine produced significant sedation and anxiolysis in comparison to those patients who were pre-medicated with oral midazolam. Therefore, all parameters indicated that preanaesthetic medication with oral clonidine may be superior to oral midazolam. This result corroborates with the findings of similar study conducted by Paris Andrea [2009].

\section{DISCUSSION}

Clonidine, an imidazoline derivative is a selective $\alpha 2$ adrenergic receptor agonist. It is a potent antihypertensive drug. It produces a fall in heart rate and blood pressure associated with decreased cardiac output but unchanged peripheral resistance. Clonidine is rapidly absorbed after oral administration and bioavailability is almost $100 \%$. Peak plasma concentration develops within 60-90 minutes after an oral dose. The plasma half-life varies from 12-24 hours. The duration of hypotensive effect after a single oral dose is about 6-12 hours. Approximately $50 \%$ of the administered drug is metabolised in the liver whereas the rest is excreted unchanged in urine. Midazolam, an imidazo-benzodiazepine derivative, is having anxiolytic, hypnotic, anticonvulsant, muscle relaxant, and anterograde amnesic effects. In normal humans, midazolam produces reductions in systolic (5\%) and diastolic $(10 \%)$ blood pressure, and increase in heart rate (18\%). Midazolam is absorbed rapidly and completely after oral administration. Maximum plasma concentrations are reached within 60-90 minutes after an oral dose. Due to substantial first-pass effect, absolute bioavailability is $30-50 \%$. $60-80 \%$ of the dose is excreted in the urine as $\alpha$-hydroxy midazolam. In young healthy adults, the elimination half-life is between 1.5 and 2.5 hours. 
The increased sympathetic activity caused by stimulation of upper respiratory tract has been supported by the observation that increase in arterial pressure during endotracheal intubation is associated with an increase in plasma noradrenaline level. ${ }^{10}$ After intubation there is gradual return of blood pressure and pulse rate to pre-laryngoscopic value due to fatigue of the receptor. Various methods have been used to attenuate the cardiovascular responses to laryngoscopy and endotracheal intubation. The effects of intravenous and oral practolol in hypertensive patients showed a significant attenuation of cardiovascular responses following laryngoscopy and intubation. ${ }^{11}$ The effect of Esmolol, a ultrashort acting beta blocker introduced in the late eighties, with an elimination half-life about 9 minutes was found to be much satisfactory so far the attenuation of cardiovascular responses to laryngoscopy and intubation are concerned. ${ }^{12}$ Intravenous lignocaine $1.5 \mathrm{mg} / \mathrm{Kg}$ administered 90 seconds before laryngoscopy and viscous lidocaine $25 \mathrm{ml}$ [4\%] given as mouth wash 12 minutes before laryngoscopy were found to be equally protective. ${ }^{4,13}$ The sedative/anaesthetic sparing properties of alpha 2 adrenergic agonists due to their actions on locus ceruleus. The brain stem nucleus that regulates a wide variety of physiological process including sleep-wakefulness regulation is inhibited by alpha 2 adrenergic agonists via a G protein mediated mechanism that involves inhibition of adenylate cyclase. M Tanaka and T Nishikawa [1994] ${ }^{14}$ studied the effect of oral clonidine premedication in attenuating hypertensive response to ketamine. Rudra A, Das AK and Chaudhari S [1994] ${ }^{15}$ studied the evaluation of clonidine as a premedicant during ketamine anaesthesia and revealed that, clonidine provide acceptable level of sedation, similar anxiolysis as diazepam ( $p>0.1$ ), good antisialagogue effect and better cardiovascular stability.

K Mikawa, K Nishina, Y Takao et al [1995] ${ }^{16}$ concluded that high oral dose of clonidine $[4 \mathrm{mcg} / \mathrm{Kg}]$ causes significant attenuation of haemodynamic and catecholamine changes compared to other two groups by suppression of increased sympathetic activity evoked by intubation. Kikuchi K, Konishi A, Watanabe Y. [1995] ${ }^{17}$ concluded that oral midazolam is an effective preanesthetic medication in adult. Ahmed N, Khan FA $[1995]^{18}$ studied for evaluation of oral midazolam as premedication in day care surgery in adult Pakistani patients. They indicated that rapidly acting oral midazolam in doses of $7.5 \mathrm{mg}$ provided safe and effective premedication in terms of anxiolysis, sedation, amnesia and psychomotor performance and is suitable for day surgery. Dipak K Raval et al. [2002] ${ }^{19}$ showed clonidine produced marked sedation and better anxiolysis as compared to placebo but less sedation and same level of anxiolysis as compared to diazepam.

Mrinmoy Das, Manjushree Ray and Gauri Mukherjee [2007 $]^{20}$ found in their study that clonidine has been shown to reduce perioperative haemodynamic instability. The aim of the study was to investigate the clinical efficiency of oral clonidine premedication in prevention of haemodynamic response associated with pneumoperitoneum. Pradipta Bhakta, B. R. Ghosh, Manjushree Roy and Gouri Mukherjee [2007] ${ }^{21}$ conducted a study for evaluation of intranasal midazolam for preanaesthetic sedation in paediatric patients. They concluded that $0.2 \mathrm{mg} / \mathrm{Kg}$ intranasal midazolam is an effective method of producing anxiolysis and sedation in paediatric patients. Paris, Andrea ${ }^{22}$ [2009] conducted a study for effects of clonidine and midazolam premedication on Bispectral index and recovery after elective surgery. They took, in a randomized, double-blind study, effects of clonidine (150 $\mu \mathrm{g}$ orally), midazolam (7.5 mg orally) and placebo administered 60-90 min prior to estimated anaesthesia induction time were investigated in 60 healthy ASA I or II patients.

They indicated that clonidine augmented haemodynamic stability and partially blunted stress responses as determined by adrenocorticotropic hormone plasma levels. In addition, clonidine did not delay postoperative recovery. Therefore, surrogate parameters indicated that preanaesthetic medication with clonidine may be superior to midazolam in healthy individuals. Oral midazolam as a premedicant was able to attenuate the pulse rate in a better way than oral clonidine. But it reduced respiratory rate significantly too. It was also found that oral clonidine produced significant sedation and anxiolysis in comparison to oral midazolam.

\section{CONCLUSIONS}

Preanaesthetic medication forms an integral part of anaesthetic management. From the present study it could be concluded that oral clonidine is the better attenuator of cardiovascular responses to laryngoscopy and intubation. Oral clonidine produced significant sedation and anxiolysis in comparison to oral midazolam. Therefore, oral clonidine could be regarded as drug of choice as a premedicant among the two drugs studied here.

\section{REFERENCES}

[1] King BD, Harris LC, Griefenstein FF. Reflex circulatory responses to direct laryngoscopy and tracheal intubation performed during general anaesthesia. Anesthesiology 1951;12 (5):556-66.

[2] Prys-Roberts C, Greene L, Miloche R, et al. Studies of anaesthesia in relation to hypertension. II. Haemodynamic consequences of induction and endotracheal intubation. Br J Anaesth 1971;43 (6):53147.

[3] Denlinger JK, Ellison N, Ominaky AJ. Effects of intratracheal lidocaine on circulatory responses to tracheal intubation. Anesthesiology 1974;41 (4):409-12.

[4] Stoelting RK. Blood pressure and heart rate changes during short duration of laryngoscopy for tracheal intubation: influences of viscous or intravenous lidocaine. Anesth Analg 1978;57 (2):197-9.

[5] Martin DE, Rosenberg H, Ankburg SJ, et al. Low dose fentanyl blunts the circulatory response to tracheal intubation. Anesth Analg 1982;61 (8):680-4.

[6] Black TE, Kay B, Healy TE. Reducing the haemodynamic responses to laryngoscopy and intubation. A comparison of alfentanil with fentanyl. Anaesthesia 1984;39 (9):8837. 
[7] Figueredo E, Garcia-Fuentes EM. Assessment of the efficacy of esmolol on the haemodynamic changes induced by laryngoscopy and tracheal intubation: a metaanalysis. Acta Anaesthesiol Scand 2001;45 (8):1011-22.

[8] Sessler CN, Grap MJ, Ramsay MA. Evaluating and monitoring analgesia and sedation in the intensive care unit. Crit Care 2008;12 (Suppl 3):S2.

[9] Hamilton M. The assessment of anxiety states by rating. Br J Med Psychol 1959;32 (1):50-5.

[10] Russell WJ, Morris DB, Frewin DB, et al. Changes in plasma catecholamine concentration during endotracheal intubation. Br J Anaesth 1981;53 (8):873-9.

[11] Prys-Roberts C, Foex P, Brio GP, et al. Studies of anaesthesia in relation to hypertension. V. Adrenergic beta receptor blockade. Br J Anaesth 1973;45 (7):671-81.

[12] Achola KJ, Jones MJ, Mitchell RW, et al. Effects of betaadrenoceptor antagonism on the cardiovascular and catecholamine responses to tracheal intubation. Anaesthesia 1988;43 (6):433-6.

[13] Maze M, Tranquili W. Alpha-2 adrenoceptor agonists: defining the role in clinical anesthesia. Anesthesiology 1991;74 (3):581-605.

[14] Tanaka M, Nishikawa T. Oral clonidine premedication attenuates the hypertensive response to ketamine. $\mathrm{Br} \mathrm{J}$ Anaesth 1994;73 (6):758-62.
[15] Rudra A, Das AK, Chaudhari S. Evaluation of clonidine as a premedicant during ketamine anaesthesia. Journal Anaesthesiology and Clinical Pharmacology 1994;11 (2):123-7.

[16] Mikawa K, Nishina K, Maekawa N, et al. Attenuation of the catecholamine response to tracheal intubation with oral clonidine in children. Can J Anesth 1995;42 (10):869-74.

[17] Kikuchi K, Konishi A, Watanabe Y. Efficacy of oral midazolam as premedication in adult. Masui 1994;43 (12):1824-7.

[18] Ahmed N, Khan FA. Evaluation of oral midazolam as premedication in day care surgery in adult Pakistani patients. J Pak Med Assoc 1995;45 (9):239-41.

[19] Raval DL, Mehta MK. Oral clonidine pre medication in attenuation of haemodynamic response to laryngoscopy and intubation. Indian J Anaesth 2002;46 (2):124-9.

[20] Das M, Ray M, Mukherjee G. Haemodynamic changes during laparoscopic cholecystectomy: effect of clonidine premedication. Indian J Anaesth 2007;51 (3):205-10.

[21] Bhakta P, Ghosh BR, Roy M, et al. Evaluation of intranasal midazolam for pre-anaesthetic sedation in paediatric patients. Indian J Anaesth 2007;51 (2):111-6.

[22] Paris A, Kaufmann M, Tonner PH, et al. Effects of clonidine and midazolam premedication on bi-spectral index and recovery after elective surgery. Eur J Anaesthesiol 2009;26 (7):603-10. 